\title{
Short-term outcome of endoscopic versus microscopic pituitary adenoma surgery in a single center.
}

\author{
Deyan Popov*, Asen Hadzhiyanev, Asen Bussarsky, Dilyan Ferdinandov \\ Clinic of Neurosurgery, "St. Ivan Rilski” University Hospital, Medical University of Sofia, “Acad. Ivan Geshov" Str, \\ Sofia, Bulgaria
}

\begin{abstract}
Pituitary Adenomas (PA) are a group of benign tumors with diverse biological behavior including different hormonal secretion, cavernous sinus invasion and considerable rates of relapse. The aim of the study was to evaluate the benefits of the Endoscopic Endonasal Transsphenoidal (EET) approach for managing hormonal producing Pituitary Adenomas (PA) in terms of achieving clinical remission in comparison to the Microscopic Endonasal Transsphenoidal (MET) approach. During the period 2014 to 2017 a series of 128 patients with PA were operated on via MET $(n=62)$ and EET $(n=66)$ approach. Endocrinological, neuro-ophthalmic and MRI examinations were assessed preoperatively and postoperatively at $3^{\text {rd }}$ and $12^{\text {th }}$ month. Thirty-two patients were diagnosed with growth-hormone secreting adenomas (GH), 25 with Adrenocorticotropic Hormone secreting adenomas (ACTH), 17 with Prolactin-secreting adenomas (Prl) and 54 with non-secreting pituitary adenomas. The endoscopic group had higher levels of remission in comparison to the microscopic group (81.8\% vs. 70.9\%), however no significant difference was observed $(\mathbf{p}=\mathbf{0 . 1 2 8})$. PA with invasiveness to the cavernous sinus from the endoscopic group had significantly better remission rates opposed to the microscopic group $(p<0.05)$. Macroadenomas not exceeding $\mathbf{4 0} \mathrm{mm}$ in diameter had higher remission rates in the endoscopic group $(\mathbf{p}<0.05)$. Examination of postoperatiove complications showed similar incidence of postoperative diabetes insipidus and CSF leak $(p>0.05)$. The use of endoscopic endonasal transphenoidal approach provides better remission rates in invasive PA compared to the conventional microsurgical approach. A long-term follow-up is still needed for further evaluation of our results.
\end{abstract}

Keywords: Pituitary adenomas, Endoscopic, Microscopic.

Accepted on July 10, 2018

\section{Introduction}

Pituitary adenomas represent a group of tumors with a diverse biological behavior. Series of studies showed that in around $16 \%$ of the population asymptomatic incidental pituitary adenomas are found in autopsy and Magnetic Resonance Imaging (MRI) studies. Some of those tumors are symptomatic and those adenomas have been estimated to be $94 \pm 19.3$ cases/ 100,000 population [1]. Symptomatic PA are considered to be $7 \%-17 \%$ of all intracranial tumors. Pituitary adenomas arise from adenohypophyseal cells in the anterior pituitary, and despite their benign histologic nature, they may enlarge and invade surrounding structures [1].

In the late 1960s Hardy popularized the use of the operating microscope in transsphenoidal surgery for selective adenomectomy. In the following 30 years, the microscopic transsphenoidal procedure via a sublabial or endonasal approach became the "gold standard" for surgically treating pituitary adenomas [2]. Jankowski introduced for the first time in 1992 a successful endonasal endoscopic adenomectomy in 3 patients [3]. The first clinical series of 50 patients, who underwent a pure endoscopic pituitary surgery for adenomas without the use of a microscope was presented by Jho and Carrau [4] in 1997. Since then, endoscopic pituitary surgery has gained great popularity, and many microscopic pituitary surgeons have transitioned to an endoscope-assisted method or fully endoscopic transsphenoidal approach for pituitary adenomas and other parasellar tumors [4].

The endoscope has some advantages over the microscope in pituitary adenoma removal, which are the enhanced visualization and better illumination. The improved panoramic high-resolution view can lead theoretically to better tumor removal in comparison to the tunnel vision and relatively restricted access of the microscope [5].

\section{Material and Methods}

During the period 2014 to 2017 a series of 128 patients with PA were operated on via MET $(n=62)$ and EET $(n=66)$ approach by the same surgical team. 


\section{Subjects}

This prospective study enrolled 128 patients with functioning and non-functioning pituitary adenomas-54 males and 74 females at average age of $48.7 \pm 15.2$ (20-77 y), referred for pituitary surgery to the Clinic of Neurosurgery of "St. Ivan Rilski” University Hospital in Sofia between 2014 and 2017.

All patients from both groups were additionally divided into smaller subgroups, according to secretion (prolactinomas, somatotropinomas, corticotropinomas and non-secreting adenomas) and according to size of tumor (micro- and macroadenoma). 14 patients from the EET group and 12 from the MET group were previously operated.

\section{Pre- and post-operative imaging and endocrine evaluation}

All patients underwent pituitary MRI with and without gadolinium prior to surgery. Dynamic MRI was performed specifically in the setting of functioning microadenomas with no adenoma visualized on conventional pituitary MRI. Hormonal data included pre- and postoperative levels of serum prolactin, morning serum cortisol and ACTH levels, $24 \mathrm{~h}$ urinary free cortisol levels and dexamethasone suppression test (for patients with Cushing disease), GH levels, IGF-1 levels, free or bioavailable testosterone levels, thyroxine levels, and TSH levels. Diagnosis and clinical remission in functioning pituitary adenomas were defined according to latest consensuses and guidelines, combined with the data of the preand postoperative MRI imaging at $3^{\text {rd }}$ and $12^{\text {th }}$ month postop. For non-functioning pituitary adenomas absence of residual tumor on the postoperative imaging studies was assessed as clinical remission. Patients underwent complete neuroophthalmic examination prior to the surgery and postoperatively at $3^{\text {rd }}$ and $12^{\text {th }}$ month.

\section{Surgical methods}

Direct microscopic mononastril endonasal transsphenoidal adenomectomy: The direct endonasal approach is performed with no septal dissection and the nasal speculum is inserted into the nostril and opened at the anterior wall of the sphenoid sinus between the two ostia after subluxing the septum and exposing the face of the sphenoid. After the anterior sphenoidotomy, opening of the sellar floor and dura are done the tumor is removed using suction, pituitary rongeurs, and various angled ring curettes [6,7].

Monoportal and biportal endoscopic endonasal adenomectomy: Preoperatively the nasal cavity is packed with cotonoids, soaked in mixture of lidocaine $1 \%$ and epinephrine $(1: 100,000)$ in order to decongest the nasal mucosa. After inspection of the nasal cavity the middle turbinate is visualized and lateralized (kept intact), and the choana and sphenoid ostia are identified. Bipolar cautery is used to dissect the posterior nasal mucosa, and to create a mucosal vascularized nasoseptal flap when needed. The septum is lateralized to the contralateral side to expose the vomer and visualize the four sphenoid ostia on each side. A sphenoidotomy and removal of the sphenoid septa is created using a high speed drill with $4 \mathrm{~mm}$ diamond bit. If a biportal approach is chosen an additional mucosal dissection of the posterior nasal mucosa is performed in the other nostril. At this point the operation can be executed using a two-hand technique (one surgeon) or a four-hand technique, using the biportal approach. The adenoma is removed using various angled curettes, pituitary rongeurs, and suction. Following the resection of the lesion, a visual inspection of the resection cavity is performed using a 30- and 45-degree endoscope to visualize compartments outside of the direct line of view. An endoscopic hydroscopy of the resection cavity was also performed. The sellar space was patched either with an autologous fat graft, hemostatic cellulose polymer, dural sealant, fibrin glue and/or nasoseptal flap if needed. Intrasellar hydroscopy was performed in 51 cases of all operated in the EET group.

\section{Statistical analysis}

The statistical analysis was performed using SPSS for Windows, Version 23.0. (SPSS Inc, Chicago, USA). For data analysis, the following statistical methods were used: descriptive statistics for tabular and graphical presentation of results, Chi-square test, Fisher exact test and Mann-Whitney U test. The obtained results were assessed as statistically reliable in threshold level of significance $\mathrm{p}<0.05$.

\section{Results}

\section{Study population}

There were 62 patients ( 33 female and 29 male) in the MET group and 66 patients in the EET (41 female and 25 male) group. Avarage age was $49.44 \pm 14.98$ and $47.90 \pm 15.70$ years and mean tumor size was $4248.62 \pm 6985.38 \mathrm{~mm}^{3}$ and 4391.04 $\pm 6400.59 \mathrm{~mm}^{3}$ in MET and EET group respectively. No statistical difference was observed between the 2 groups, particularly with regard to tumor volume, type of adenoma and rate of cavernous sinus invasion $(\mathrm{p}>0.05)$.

In the microsurgical group an overall remission of $70.97 \%$ was achieved. The subgroups of patients with prolactinomas $(n=8)$, somatotropinomas $(n=15)$, corticotropinomas $(n=14)$ and nonsecreting adenomas $(\mathrm{n}=25)$ had a remission rate of $75 \%$, $66.6 \%, 85.7 \%$ and $64 \%$ respectively. Remission in the subgroups of patients with microadenomas and macroadenomas was $88.46 \%$ and $58.3 \%$ respectively (Table 1 ). The mean length of hospital stay was $6.34 \pm 2.17 \mathrm{~d}$. The mean duration of the surgeries was $86.85 \pm 21.29 \mathrm{~min}$.

The endoscopic group had an overall remission of $81.82 \%$. The subgroups of patients with prolactinomas $(n=9)$, somatotropinomas $(n=17)$, corticotropinomas $(n=11)$ and nonsecreting adenomas $(n=29)$ had a remission rate of $88.89 \%$, $81.82 \%, 82.35 \%$ and $79.31 \%$ respectively. Remission in the subgroups of patients with microadenomas and macroadenomas was $88 \%$ and $78 \%$ respectively (Table 1 ). The mean length of hospital stay was $5.79 \pm 1.81 \mathrm{~d}$. The mean duration of the surgeries was $113.48 \pm 18.52 \mathrm{~min}$. 


\section{Comparison between MET and EET group}

There was no statistical difference between the remission rates in both groups in functioning and non-functioning PA, as well as between microadenomas and macroadenomas $(\mathrm{p}>0.05)$. Further breakdown of this data in terms of size, revealed that in the subgroup macroadenomas, excluding the giant macroadenomas (macroadenomas above $40 \mathrm{~mm}$ ), there were significantly better remission rates in the endoscopic group in comparison to the microscopic group $(p=0.043)$. In the MET group there was significant difference between remission rates of microadenomas and macroadenomas $(p=0.01)$. This phenomenon was not observed in the EET group $(\mathrm{p}>0.05)$. Further investigation showed that the distribution of tumor volumes across the categories with remission and without remission was the same in the EET group $(\mathrm{p}>0.05)$ and was significantly different in the MET group $(\mathrm{p}=0.030)$. In invasive PAs (Knosp scale above 0 ) there were better remission rates in the group, treated trough EET approach $(\mathrm{p}=0.025)$.

\section{Complications}

CSF leak rates were similar in the two groups. Five patients $(8.06 \%)$ in the MET group and $7(10.6 \%)$ in the EET group had post-operative CSF leakage (Table 2). Meningitis, diabetes insipidus, postoperative hypopituitarism had similar rates and no statistical difference between both groups was found $(\mathrm{p}>0.05)$.

Table 1. Remission rates in patients in microsurgical (MET) and endoscopic (EET) group.

\begin{tabular}{|c|c|c|c|c|c|}
\hline & MET remission (n) & MET remission (\%) & EET remission (n) & EET remission (\%) & $P$ value \\
\hline All PAs & $44 / 62$ & $70.97 \%$ & $54 / 66$ & $81.82 \%$ & $p>0.05$ \\
\hline Prolactinomas & $6 / 8$ & $75 \%$ & $8 / 9$ & $88.89 \%$ & $p>0.05$ \\
\hline Corticotropinomas & $12 / 14$ & $85.71 \%$ & $9 / 11$ & $81.82 \%$ & $p>0.05$ \\
\hline Somatotropinomas & $10 / 15$ & $66.67 \%$ & $14 / 17$ & $82.35 \%$ & $p>0.05$ \\
\hline Non-functioning PAs & $16 / 25$ & $64 \%$ & $23 / 29$ & $79.31 \%$ & $p>0.05$ \\
\hline Microadenomas & $23 / 26$ & $88.46 \%$ & $22 / 25$ & $88 \%$ & $p>0.05$ \\
\hline Macroadenomas & $21 / 36$ & $58.33 \%$ & $32 / 41$ & $78.05 \%$ & $p>0.05$ \\
\hline
\end{tabular}

Table 2. Postoperative complications in MET and EET group.

\begin{tabular}{|c|c|c|c|c|c|}
\hline & MET (n) & MET (\%) & EET (n) & EET (\%) & $P$ value \\
\hline CSF & 5 & 8.06 & 7 & $10.60 \%$ & $p>0.05$ \\
\hline Epistaxis & 1 & 1.6 & 1 & $1.50 \%$ & $p>0.05$ \\
\hline $\begin{array}{l}\text { Transitory } \\
\text { insipidus }\end{array}$ & 10 & 16.12 & 12 & $18.18 \%$ & $p>0.05$ \\
\hline $\begin{array}{l}\text { Permanent } \\
\text { insipidus }\end{array}$ & - & - & 1 & $1.50 \%$ & $p>0.05$ \\
\hline Death & - & - & - & - & $p>0.05$ \\
\hline Hypopituitarism & 7 & 11.29 & 8 & $12.12 \%$ & $p>0.05$ \\
\hline
\end{tabular}

\section{Discussion}

The microscopic transsphenoidal approach has been the most common technique for resecting pituitary lesions over the last 50 years. Endoscopic transsphenoidal surgery for pituitary adenomas was introduced in the 90s and it has been developing rapidly over the last 20 years with introduction of new techniques. There are a couple of meta-analysis and retrospective series suggesting endoscopic techniques are associated with achieving gross total removal in pituitary tumors, especially when these tumors are locally invasive or are not just limited to the sella [8-11]. A recent meta-analysis from Almutairi including over 8000 patients showed evidence for higher gross total resection in patients, operated endoscopically. Endoscopic surgery for non-functioning PA also is associated with higher rates of GTR. However, there is no evidence that functioning PAs benefit from the endoscopic approach, as there is no statistical difference between the two surgical techniques [10].

There are few studies, which perform a volumetric analysis of the surgical results between EET and MET approach. The current shows that remission rates in patients, treated endoscopically, do not decrease with increasing volume of the PA. However increasing tumor volume leads to decreased remission rates in the microscopic group. This observation was further confirmed by statistically significant difference in the microscopic group between remission rates in the subgroups microadenoma and macroadenoma, which was not observed in the endoscopically treated group. All this data could suggest that the EET approach potentially leads to higher remission rates in macroadenomas, especially with suprasellar extension. Messerer et al. [12] also suggest that the endoscopic endonasal transsphenoidal surgery leads to better results in terms of gross total resection for macroadenomas.

Dhandapani et al. [13] compared remission rates between MET and EET approach in surgical treatment of invasive PAs to the cavernous sinus. Endoscopic endonasal transsphenoidal surgery leads to greater gross total resection in invasive PAs in comparison to the microscopic approach, probably because of the angled endoscopic view of the laterally positioned cavernous sinus. Our study also confirmed this conclusion. 
Most of the retrospective studies and meta-analysis found no difference in terms of postoperative complications, which was also observed in our study. Ammirati et al. [14] found higher rate of vascular lacerations of the internal carotid artery in patients treated endoscopically in comparison to the microscopic approach.

\section{Conclusion}

In conclusion, the results of our study show no statistical difference between the remission rates in EET and MET groups in functioning and non-functioning PA, as well as between microadenomas and macroadenomas. Endoscopic endonasal transsphenoidal approach is associated with better remission rates in surgical treatment of invasive pituitary adenomas. Both surgical methods offer similar safety profile in terms of operative and postoperative complications.

\section{Acknowledgment}

None

\section{References}

1. Asa L, Ezzat S. The cytogenesis and pathogenesis of pituitary adenomas. Endocr Rev 1998; 19: 798-827.

2. Hardy J. Surgery of the pituitary gland, using the transsphenoidal approach. Comparative study of 2 technical methods. Union Med Can 1967; 96: 702-712.

3. Jankowski R, Auque J, Simon C, Marchal C, Hepner H, Wayoff M. Endoscopic pituitary tumor surgery. Laryngoscope 1992; 102: 198-202.

4. Jho D, Carrau L. Endoscopic endonasal transsphenoidal surgery: experience with 50 patients. J Neurosurg 1997; 87: 44-51.

5. Cappabianca P, Kelly F, Laws R. Endoscopic transnasal versus open transcranial cranial base surgery: the need for a serene assessment. Neurosurgery 2008; 63: 240-243.

6. Griffith B, Veerapen R. A direct transnasal approach to the sphenoid sinus. Technical note. J Neurosurg 1987; 66: 140-142.

7. Buchfelder M, Fahlbusch R. The classic transsphenoidal approach for resection of pituitary tumors. Oper Tech Neur 2002; 5: 210-217.
8. Karppinen A, Kivipelto L, Vehkavaara S, Ritvonen E, Tikkanen E. Transition from microscopic to endoscopic transsphenoidal surgery for nonfunctional pituitary adenomas. World Neurosurg 2015; 84: 48-57.

9. Razak A, Horridge M, Connolly J, Warren J, Mirza S. Comparison of endoscopic and microscopic transsphenoidal pituitary surgery: early results in a single centre. Br J Neurosurg 2013; 27: 40-43.

10. Almutairi R, Muskens I, Cote D. Gross total resection of pituitary adenomas after endoscopic vs. microscopic transsphenoidal surgery: a meta-analysis. Acta Neurochir (Wien) 2018; 10: 1007.

11. Gao Y, Zhong C, Wang Y. Endoscopic versus microscopic transsphenoidal pituitary adenoma surgery: a metaanalysis. World J Surg Oncol 2014; 12: 94.

12. Messerer $M$, De Battista $C$, Raverot $G$, Kassis S, Dubourget J. Evidence of improved surgical outcome following endoscopy for nonfunctioning pituitary adenoma removal. Neurosurg Focus 2011; 30: 11.

13. Dhandapani S, Singh H, Negm H. Cavernous sinus invasion in pituitary adenomas: systematic review and pooled datameta-analysis of radiologic criteria and comparison of endoscopic and microscopic surgery. World Neurosurg 2016; 96: 36-46.

14. Ammirati M, Wei L, Ciric I. Short-term outcome of endoscopic versus microscopic pituitary adenoma surgery: a systematic review and meta-analysis. J Neurol Neurosurg Psychiatry 2013; 84: 843-849.

\section{*Correspondence to}

Deyan Popov

Clinic of Neurosurgery

“St. Ivan Rilski” University Hospital

Medical University of Sofia

Bulgaria 